\title{
Discussion on Maintenance Measures and Practical Application of Computer Practical Detection
}

\author{
Lin Zhou \\ Chongqing Business Vocational College, Chongqing, 401331, China
}

Keywords: computer; practical detection; maintenance

\begin{abstract}
With continuous improvement of modern people's living level, the computer has widely entered into people's home and has become a tool which is often used. However, various kinds of fault may happen in operation process of computer, and the fault diagnosis and maintenance is difficult; therefore, it is extremely necessary to be familiar with practical detection and practical maintenance measures of applied computer. This paper makes a discussion from 3 aspects, that is, judgment on false and true fault, maintenance measures for practical detection of computer hardware, and maintenance measures for practical detection of computer software. Nowadays, with wider and wider application of computer technology, the computer has become a daily necessary tool for many enterprises and individuals. However, in the process of computer application, various kinds of fault often happen. Therefore, the computer users shall learn the basic detection and maintenance measures. Despite of diversified computer fault diagnosis and maintenance methods, the author thinks that it is able to detect the fault reason through following the diagnosis principle of computer fault and careful analysis, and then it is able to let the computer provide more convenience for modern people's learning, work, and life through timely maintenance.
\end{abstract}

\section{Judgment measures on false and true fault}

As for computer faults which commonly happen, most of them are not true computer hardware fault, but software fault or false fault caused due to setting error. In view of this, to know following false computer fault phenomena can be good for saving maintenance personnel's time and quickly repairing the computer. Firstly, we shall carefully check power line, socket, switch, and various external connecting lines of computer. Once the fall-off or bad contact happens in various kinds of power lines, data lines, sockets, and switches, the computer equipment will be under abnormal operation. Therefore, we shall carefully check whether the equipment power of computer is normal, whether the plug and socket have good contact, whether the data and control lines between parts are correctly and reliably connected, and whether there exists looseness. Secondly, we shall carefully check new features of computer and the problems which may happen in setting. In fact, large quantity of so-called faults are caused due to the fact that the users are unfamiliar with new features of hardware or operation system, as well as setting problem. For example, the setting of display and hard disk jumper just belong to this situation. Therefore, we shall get more knowledge of new features of host, peripheral, and application software of computer so as to help us to eliminate various kinds of false faults. Thirdly, we shall judge false and true faults. Among computer faults, the faults caused due to virus and software damage, incorrect deletion, and misoperation, etc. account for a very high proportion. Some faults are not true faults, and we can use CMOS for judgment. The main practice is to check a jumper with 3 feet near main board CMOS battery of computer; in case of short circuit of 1 and 2, the computer is normal; in case of short circuit of 2 and 3 , it is required to clear up CMOS, that is, taking down the jumper wire of 1 and 2 and realizing short circuit of 2 and 3, and then placing the jumper wire back for 1 and 2; in this way, the computer can be normally started, which also proves that the abovementioned fault belongs to false fault.

\section{Maintenance measures for practical detection of computer hardware}

The first method is observational detection method. The first step is asking. The maintenance personnel shall inquire about the condition while the fault happens, mainly including site 
environment upon occurrence of fault, and the thing that the user is doing. For example, what sound the user hears, what smell the user smells, whether the voltage suddenly rises or it is not stable at that time, whether the lightning stroke happens and whether the user has shaken computer, etc. The second step is seeing. The maintenance personnel shall observe whether the insertion and connection of board and card is inclined, whether the lead foot of various components of computer collide with each other, whether the computer surface is burnt, whether the surface of computer chip is cracking, whether the copper foil on computer board card is burnt out, whether the foreign matter which drops into components and parts of computer main board results in short circuit, whether there is burning place on main board and card of computer, and whether the fracture has happened in lead line of printed circuit board of computer. The third step is listening. The maintenance personnel shall monitor whether the operation sound of power fan, CPU fan, hard disk motor, and display of computer is normal. Meanwhile, once the short-circuit fault happens in computer system, the abnormal sound will happen. Through monitoring, it is able to timely find the hidden danger of partial accidents, and help maintenance personnel to timely adopt effective measures to solve the problem prior to occurrence of accident. The fourth step is smelling. The maintenance personnel can smell whether there exists burning smell in various kinds of plug-in cards on host and main board for convenience of timely finding fault and determining short-circuit place. The final step is touching. The maintenance personnel can press activity chip of tube socket by hand to check whether the computer chip is loose or has bad contact. Meanwhile, while the computer system operates, the maintenance personnel can also touch or approach CPU, display, hard disk and other equipment shell of computer by hand to judge whether the computer equipments have normal operation according to the temperature. When we use hand to touch the surface of chip, it shows that the chip has been damaged in case of burning feeling.

The second method is cleaning detection method. As for those computers under poor use environment or with long continuous use time, the maintenance personnel shall firstly clean the computer. The specific step is to use brush to slightly brush away the dust on main board and peripheral of computer, and then carry out next step of inspection work. Meanwhile, because partial plug-in cards or chips in main board adopt inserting connection way, the bad contact may happen due to oxidation of lead foot caused by shaking or dust. At this time, we can use eraser to wipe off superficial oxide; after re-inserting connection, we can start the computer to check whether the fault has been eliminated.

The third method is replacement detection method. This method is one of maintenance methods in which we mainly make use of good computer part to replace the computer part for which the fault may happen so as to judge whether the computer fault will disappear. A good part basically belongs to same model; certainly, it may belong to different model. The main sequence of replacement is shown as below. Firstly, we consider the replaced part or equipment according to computer fault phenomenon. Then, we replace the part according to the sequence from easy to difficult. For example, we can firstly replace internal storage and CPU, and then replace main board. For another example, in order to judge printing fault, we can firstly consider whether there exists problem in print driver procedure of computer, and then consider whether there exists fault in print cable of computer, and then consider whether there exists fault in printer. Thirdly, we shall suspect the connecting line and signal line which connect with computer parts with fault, and then replace the computer part which is suspected of fault part, and then replace power supply part, and then other relevant parts. Finally, we shall consider the precedence order of replaced parts based on fault rate of computer parts. Under general condition, we will firstly replace computer parts with high fault rate, or insert the fault part or peripherals into other computer of same model to judge whether the computer is normal.

The fourth method is isolation detection method. This method refers to a kind of detection way of shielding partial computer hardware or computer software. This method can isolate computer hardware and software for which there exists mutual conflict so as to better judge the generation and change situation of computer fault. As for software, the shielding refers to prohibition of use and uninstalling of drive upon shutdown or uninstalling of software; as for hardware, the shielding 
refers to prohibition of use and uninstalling of drive in device manager, or direct removal of software in computer software.

The fifth method is beating detection method. This detection method is often used when the computer maintenance personnel suspect that there exists looseness in one part of computer or the computer operates under instable condition. The main implementation method is that the maintenance personnel use the fingers to slightly beat computer case shell so as to cause certain vibration, distort the position of computer equipment part, and cause appearance of fault for convenience of judging specific position of fault. If we use same method to operate 2 or above sets of same model of computers, we can observe the difference in computer with normal state and computer with fault so that we can implement preliminary judgment on corresponding fault of computer.

The sixth method is temperature rising and lowering detection method. This method is mainly operated based on basic principle of generation of computer fault, and this method can be used to detect temperature resistance performance of computer CPU and other parts and then timely eliminate the fault and hidden danger, and avoid occurrence of fault through artificially changing the temperature at computer location. While lowering the ambient environment of computer in the operation process, we can know which reason (high-temperature part or failure in bearing high-temperature parts) results in shrinkage of fault coverage based on whether the fault frequency of computer reduces. The main methods used to rise and lower the temperature at computer location include shutdown cooling, temperature control via indoor air-conditioning, electric hair drier warming and electric fan cooling, and temperature rising via long-time operation, etc.

The seventh method is program testing detection method. Although the integrated circuit has been widely applied nowadays, due to extremely complicated welding technology of integrated circuit and lack of relatively reliable data about hardware technology, it is hard to find out the fault by virtue of hardware detection; therefore, the special diagnosis card, such as computer main board diagnosis card, shall be used to detect the technical parameters of various computer parts, or the special diagnosis program is utilized to help to detect hardware parameters; in this way, it is able to obtain obvious maintenance effect. This detection method utilizes software to send data and command so as to read out abnormal data in line and find out specific position of fault.

\section{Maintenance measures for practical detection of computer software}

Firstly, the incompatibility and even conflict happens between operation system and application software of computer. If the operation environment of computer system can't be compatible with application software, it will be hard for software to operate normally, and then various kinds of instability or irregularity faults often happen. In the operation process of computer software, it is able to automatically detect the environment of computer system; if it is found incompatible via detection, the computer operation system will immediately hinder software operation. In order to ensure that the computer can keep normal operation, it is required to provide suitable system environment for software operation. Certainly, there also exists possibility of conflict between application software and application software; once the conflict happens in access area, work address and operation environment of two or more kinds of software program, it is easy to cause a mess of whole computer system, thus causing data loss or abnormal operation of software. In this way, if more software is installed in the computer, the probability of occurrence of conflict will be higher.

Secondly, the computer operation mistake results in fault. The computer operation mistake can be mainly divided into software program operation mistake and command operation mistake. After the program with destructive effect is operated or the command which shall be not executed is used, various faults such as dead halt of computer or file loss may happen. While computer users operate the computer, the operation mistake can often happen by accident. In order to avoid the occurrence of this condition as much as possible, the computer users shall pay high attention to prompts or warning in computer processing process so as to effectively avoid occurrence of error and avoid that the normal work is affected. 
Thirdly, the computer virus penetration results in fault. nowadays, with continuous development of computer network technology, the computer virus penetration phenomenon also becomes more and more serious. Once the computer procedure is maliciously invaded by virus, the virus can modify the program and destroy originally stored information of computer system; under serious situation, the normal operation of computer will be affected. The users shall timely install firewall and antivirus software to often check various kinds of virus and upgrade antivirus software so that the corresponding virus can be thoroughly checked and killed. We shall regularly scan various kinds of virus, and remove various kinds of Trojan horse program to ensure that the computer system can have a healthy, safe, and sustainable operation and development environment.

Fourthly, the network reason results in fault. The fault occurred due to computer network factor is mainly reflected at the situation that the users can't timely connect network. If the user can't surf the internet normally, it is very likely that the computer network card is damaged, the computer reticle has bad contact, and the computer network identification and user agreement can't be correctly set, etc. Therefore, we shall guarantee that the computer is not damaged and then make setting after the reticle is connected well and after correcting checking of network identification and user agreement.

\section{Conclusion}

In a word, with rapid development of science and technology in recent years, the computer technology is also greatly developed and widely applied in various industries. In order to better exert the service efficiency of computer and ensure short shutdown overhaul time, we shall timely find the fault and fault reason in using process so as to find out elimination measure; however, due to many fault reasons, the fault is often a consequence of many factors. As long as we find out the reason, we can adopt reasonable measures to shorten detection time to maximum degree.

\section{Acknowledgments}

This paper is a faculty-level project, and the project name is Discussion on Professional Education-Oriented Education Mode of Business Vocational Colleges (project No.: 2012yjkt04).

\section{References}

[1] Zhang Yumei: Discussion on Maintenance Method and Application of Computer Practical Detection [J], Modern Science, 2010 (12).

[2] Zhang Dong: Discussion on Maintenance Method and Application of Computer Practical Detection [J], Heilongjiang Science and Technology Information, 2010 (24).

[3] Chen Shilei, Li Jiwei: Discussion on Overhaul and Maintenance of Common Computer Faults [J], Public Communication of Science \& Technology, 2011 (1).

[4] Wang Xueke: Correct Use and Maintenance Methods of Home Computer [J], China Educational Technology \& Equipment, 2011 (12).

[5] Tao Chongfu: Discussion on Daily Maintenance and Handling of Common Faults of Computer Hardware [J], Computer Programming Skills \& Maintenance, 2011 (18). 